\title{
Experiments on the Use of Signal Visualization Technique for In-Service Stall Detection in Industrial Fans
}

\author{
Stefano Bianchi, ${ }^{1,2}$ Alessandro Corsini, ${ }^{1,2}$ and Anthony G. Sheard ${ }^{3}$ \\ ${ }^{1}$ Department of Mechanical and Aerospace Engineering, University of Roma-Sapienza, Via Eudossiana 18, 00184 Roma, Italy \\ ${ }^{2}$ Soluzioni Energia \& Diagnostica Srl, Via ASI Consortile 7, 03010 Ferentino, Italy \\ ${ }^{3}$ Fläkt Woods Ltd, Axial Way, Colchester, Essex CO4 5ZG, UK
}

Correspondence should be addressed to Alessandro Corsini; alessandro.corsini@uniromal.it

Received 4 October 2012; Accepted 15 March 2013

Academic Editor: Tamer Elnady

Copyright (C) 2013 Stefano Bianchi et al. This is an open access article distributed under the Creative Commons Attribution License, which permits unrestricted use, distribution, and reproduction in any medium, provided the original work is properly cited.

\begin{abstract}
The paper describes a stalldetection criterion based on the use of symmetrised dot pattern (SDP) visual waveform analysis and the stallwarning methodology based on a recently developed analysis. The experimental study explores the capability of the SDP technique to detect the stall incipience and evolution in the presence of low signal-to-noise ratios, that is, a noisy working environment. Moreover, the investigation presents a systematic analysis on the probe position's influence with respect to the fan section. As such, the SDP technique in combination with an acoustic measurement is able to create a visual pattern that one can use to detect stall from potentially any location around the fan/duct system.
\end{abstract}

\section{Introduction}

Aerodynamic instability arises when flow rate throttling constrains the operational envelope of fans, blowers, and compressors. To avoid instability, aerodynamicists must provide adequate stability (stall) margin to accommodate inlet distortions, degradation due to wear, throttle transients, and other factors that reduce fan, blower, and compressor stability from the original design baseline. Because aerodynamic stall, resulting in increased alternating aerodynamic loads, is a major potential cause of mechanical failure in axial fan stall, detection techniques have had wide application for many years. Researchers have studied the detection and analysis of the different forms of aerodynamic instability for several decades [1-4].

The standard classification of aerodynamic stalls in axial fans and compressors [5] distinguishes between rotating stall (in which reversed flow regions occur locally) and surge (which is characterised by periodic backflow over the entire annulus involving violent oscillations in the air flow which can result in mechanical failure such as fan blade breakage). The latter is unusual in fan applications due to the system's insufficient counterpressure. Rotating stall is a mechanism by which the rotor adapts to a reduction in flow rate. This results in circumferentially nonuniform flow patterns rotating in the annulus. In reviewing the evolution of rotating stall, Cumpsty [6] noted that the drop in overall performance can occur as a so-called "progressive stall" or an "abrupt stall." Engineers usually associated the former with a partspan stall which results in a small performance drop, whereas they associate the latter with a full-span stall and a large drop in performance. Notably, the part-span rotating stall occurs typically in single blade rows [6] and usually leads to more complex disturbances in single-rotor or single-stage machines than in multistage compressors [7]. All of these forms of abnormalities are even magnified in rotors operating under erosive regimes. In fact, the recovering of pressure rise capability which is the consequence of leading edge and blade surface wear is actuated by increasing the blade pitch angle which reduces the fan's stall-free operating margin. These combined factors place considerable mechanical stress on fan rotors, eventually leading to mechanical failure. Strain gauge measurements on axial compressors [8] and fans [9] have bending stress in vanes exceeding stable operation by a factor of five and seven, respectively, under "rotating stall" conditions. Reliable means of monitoring the stability limit on time are still research subjects $[10,11]$. The request of developing warning systems of general validity [12] motivates 
this circumstance and identifies stall as early as possible to enable an active control system to react and suppress the incipient stall. Early warning time, according to the literature, is approximately two rotor revolutions for axial compressors [13] but perhaps ten times longer for industrial fans [14].

Prior methods have had drawbacks in their inability to enable a sufficiently rapid response to the onset of stall to avoid damage and their inability to sense the approach to stall. First, researchers have identified alert methods for individual test beds, but reliable warnings of general validity require further research. Second, techniques for the detection of stall initiation based on experimental observation of prestall behaviours have sought to identify such behaviour as early as possible to enable an active control system to react and suppress the incipient stall. As a third factor, all the nonmodel-based detection techniques rely on the use of on-board probes able to sense the unsteady pressure evolution in the vicinity of the blade rows, that is, usually flush mounted on the casing. These nonmodel-based early warning techniques for real-time control applications are mostly based on time or Fourier analyses [15-17]. In this number, Tahara et al. [18] proposed a stall warning index (utilising pressure signals with high-response transducers on the rotor casing) by taking a normalised product of pressure unsteadiness over successive rotor revolutions. Breugelmans et al. [19] speculated on the underlying chaotic dynamics of rotating stall. They reconstructed phase-space portraits of the experimental data series, that is, velocity, static pressure, and vibration signals, using the so-called method of delays which Takens [20] proposed. In a similar vein, Bright et al. [21] investigated rotating instabilities prior to stall with "temporal structure function" inspired by a statistical approach derived from chaos theory. Stall inception mechanisms can often correlate with a specific acoustic signature. To this end, researchers have proposed diagnostic techniques based on sound-signal visualisation $[22,23]$. This approach to fault diagnosis in rotating machinery is suitable in the case of pressure signals dominated by discrete narrow and frequency components related to the rotor speed. Recently, Sheard and coauthors [24] used sound visualisation to assess the acoustic payoff in a class of low-solidity axial fans fitted with passive control of tip leakage-generated noise. Successively, Bianchi et al. [25] refined the technique and applied it as a stall warning tool in axial rotors.

The present study explores the use of sound-signal visualisation based on early stall warning detection. Whilst Bianchi et al. [25] reconstructed the symmetrised dot pattern (SDP) from the hydrodynamic pressure which they took at the rotor's casing wall, the authors of this paper developed the proposed technique using the reconstruction of SDP images from sound pressure signals. In doing so, they extended the technique to the use of measured acoustic pressure signals in different locations at the fan's acoustic far-field. The authors used the technique, which researchers originally conceived for the visual characterisation of speech waveforms and sound quality evaluation [26-28], in the early stall warning of ventilating fans [14] and proved its effectiveness in a time window lasting from 0.1 to $0.004 \mathrm{~s}$. This finding confirmed
TABLE 1: Specifications of $A C 90 / 6 / 28$ fan.

\begin{tabular}{lcc}
\hline \multirow{2}{*}{ Blade geometry } & \multicolumn{2}{c}{ AC90/6 fans } \\
& Hub & Tip \\
\hline$l / t$ & 1.32 & 0.31 \\
Pitch angle (deg) & 36 & 28 \\
Camber angle (deg) & 46 & 41 \\
Solidity & 1.24 & 0.3 \\
\hline Fan rotor & \multicolumn{2}{c}{6} \\
Blade number & \multicolumn{2}{c}{28} \\
Blade tip pitch angle (deg) & \multicolumn{2}{c}{0.22} \\
Blade tip stagger angle (deg) & \multicolumn{2}{c}{900.0} \\
Hub-to-casing diameter ratio & \multicolumn{2}{c}{1.0} \\
Tip diameter (mm) & \multicolumn{2}{c}{$935 \div 950$} \\
Rotor tip clearance (\% span) & \multicolumn{3}{c}{ Rated rotational frequency (rpm) }
\end{tabular}

the SDP potential technique as a diagnostic tool for rotating instabilities related to stall detection in fair time.

The present program of work verifies the SDP-based early stall warning technique in a test environment relevant to a cooling fan unit's in-service operation. The experimental study explores the SDP technique's capability to detect the stall incipience and evolution in presence of low signal-tonoise ratios, that is, a noisy working environment. Moreover, the investigation presents a systematic analysis on the probe position's influence with respect to the fan section. As such, the SDP technique in combination with an acoustic measurement creates a visual pattern that engineers can use to detect stall in any location, not just with the microphone over the blade itself. The work also focuses on proving the visualisation method's capability in presence of low signalto-noise ratio, that is, low instability signature-to-background noise ratio. This circumstance, to simulate operational environments, speculated on the effectiveness of visual waveform analysis with the probe located in the far-field.

\section{Experimental Methodology Outlines}

The study was performed on a family of commercially available cooling fans, coded $A C 90 / 6 / 28$. In-service experience had indicated that this family of fans gives good acoustic performance with respect to the state-of-the-art configurations. The investigated fans were equipped with a six-blade unswept rotor, with blade profiles of a modified ARA-D geometry type originally designed for propeller applications. Table 1 provides specifications of the fan $A C 90 / 6 / 28$.

2.1. Investigated Flow Conditions. The fan rotor was studied at a large tip pitch angle of 28 degrees (when measured in the peripheral direction). This constituted the customary setting for these fans. The study accounted a full performance test (Figure 1), in the way prescribed by the British Standard [29]. In summary, the experimental test matrix consisted of measuring the far-field noise during the fan transient from the normal operation point, with a total pressure rise $P_{t}=$ $125 \mathrm{~Pa}$, up to the stall margin at a total pressure rise $P_{t}=208 \mathrm{~Pa}$ 


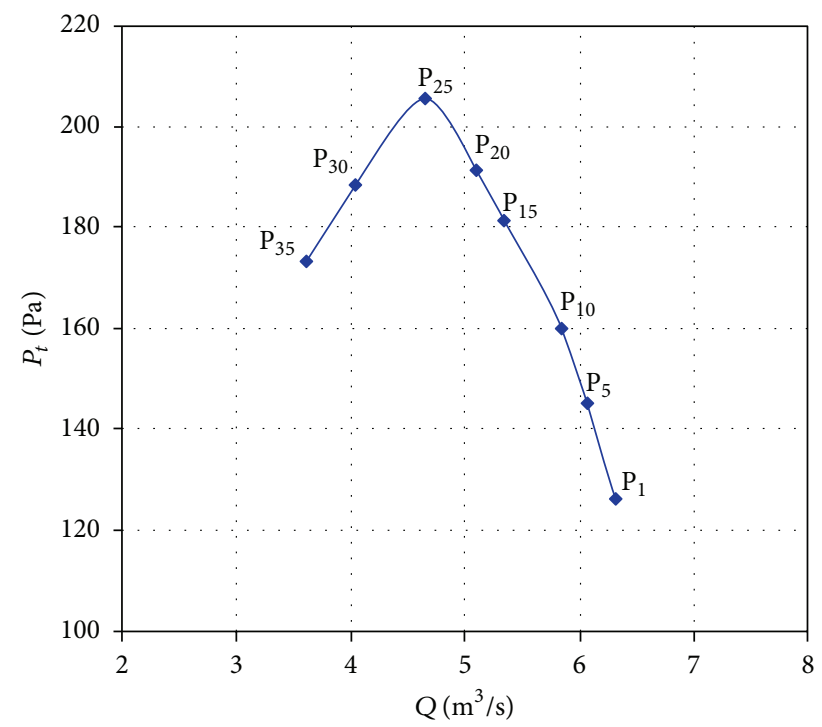

FIGURE 1: Fan operative diagram and tested operative points.

and then down to perform a full-stall. Figure 1 reports the tested operative points.

2.2. Experimental Setup. The experiments were conducted in the Latina-Borgo Isonzo Laboratory of the University of Roma, Sapienza (Italy). The fan was rigged in a fully ducted facility which certifies the performance of industrial fans according to the BS848-1.6:2000 standard, which is equivalent to the ISO 10302:1996 standard. The rotor balancing ensures vibration below $4 \mathrm{~mm} / \mathrm{s}$ threshold. The fan was operated in a custom-built casing made from rings of cast and machined steel. Clearance between the blade tips and the casing was constant at $1 \%$ of blade span. A $2.5 \mathrm{~kW}$, directcoupled induction 400-volt (AC), three-phase CM29 motor drove the rotor at a constant speed of $940 \mathrm{rpm}$. The blade tip speed was $44.34 \mathrm{~m} / \mathrm{s}$. Under these conditions, the blade passing frequencies (BPFs) for all the tested configurations were in the range $93.75-96.88 \mathrm{~Hz}$.

Noise measurements were carried out at the fan inlet (A) and outlet (B) and in two positions on the duct side: at the fan location (C) and at the inlet duct's middle length (D). All the measurements were done with the microphone $2 \mathrm{~m}$ off the duct casing. Figure 2 depicts the measurement setup. An NI Compact Acq. 9172 with NI 9205 analogical input module acquired the signals from the far-field microphone. In all cases, the authors measured whole signals for $60 \mathrm{~s}$ during three repeated measurement sessions.

In order to test the effectiveness of SDP-based instability detection techniques in a noisy environment, the authors carried out the measurement campaigns with $39 \mathrm{~dB}$ background noise. The preliminary calculation of SNR gave a range of $1.99-2.22 \mathrm{~dB}$.

\section{Stall Warning Technique}

The rationale of the proposed warning technique is to link topology and stall dynamics in order to help visualise the range of possible system behaviours. In so doing, it shares the foundation of the chaos theory [30]. The basic idea is that with visualising the shape, it is possible to understand the system, not only quantitatively (sound power level produced by tonal components), but by representing howling and modulated sound components.

The SDP pattern provides a local visual correlation that one can apply to detect the significant features of any signal. As such, the visual characterisation of speech waveforms was conceived in automatic human voice recognition algorithms [26]. The merit of the methodology is its ability to perceive otherwise "unquantifiable" differences in sound signals mimicking the way that humans hear $[27,28]$.

\subsection{Mathematical Framework and Polar Pattern Construction.} The SDP polar transformation requested the reading of a pressure signal with a constant delay time, called time lag, in a similar fashion to the phase-space portrait reconstruction method [20]. The sampled data set were used to construct two vectors, representing the two-dimensional coordinates of a polar mapping, with the second vector of data differing from the first because of the initial shifting in time of the adopted lag. An algorithm that maps a normalised time waveform into symmetrised dot patterns on a polar graph produces SDPs. Figure 3 shows the SDP plotting technique.

A point in the time waveform maps onto a radial component and the adjacent point maps to an angular component. The polar transformation $R(i)$ from waveform to SDP reads as follows:

$$
\begin{gathered}
R(i)=\frac{\sigma(i)-\sigma_{\min }}{\sigma_{\max }-\sigma_{\min }}, \\
\Theta^{+}(i)=\Theta_{0}+\frac{\sigma(i+L)-\sigma_{\min }}{\sigma_{\max }-\sigma_{\min }} \xi, \\
\Theta^{-}(i)=\Theta_{0}-\frac{\sigma(i+L)-\sigma_{\min }}{\sigma_{\max }-\sigma_{\min }} \xi,
\end{gathered}
$$

in which $i$ is the number of $\operatorname{dot}(i=\operatorname{integer}(t / \Delta t))$, with $t$ the time abscissa and $\Delta t$ the sampling time; $L$ is the time lag coefficient; $\sigma(i)$ is the sampled $i$ th sound signal; $\sigma_{\max }$ and $\sigma_{\min }$ are the highest and the lowest value of the original waveform window; $\Theta_{0}$ is the rotation of the origin angle of any reference line; $\xi$ is the angular gain of the plotting, and $\Theta^{+}$and $\Theta^{-}$are the two angles of the traditional polar space.

The input waveform first normalises in the range $(0, \xi)$ by finding the higher $\left(\sigma_{\max }\right)$ and lower values $\left(\sigma_{\min }\right)$ for the $N$ data points in the window. Overall amplitude is therefore, in general, not a factor in the characterisation. By creating a scatter plot of neighbour amplitudes on polar coordinates, the symmetrised dot pattern space is able to discriminate the frequency of the time signals and their variability.

In plotting SDP patterns, the methodology takes the following parameters: (i) time lag $L$ and (ii) the angular gain $\xi$. These parameters influence the topological aspect of the polar mapping and the ability to identify the aerodynamic phenomena of interest.

In view of the verification of the early stall warning capability, it is also important to consider the role of the 


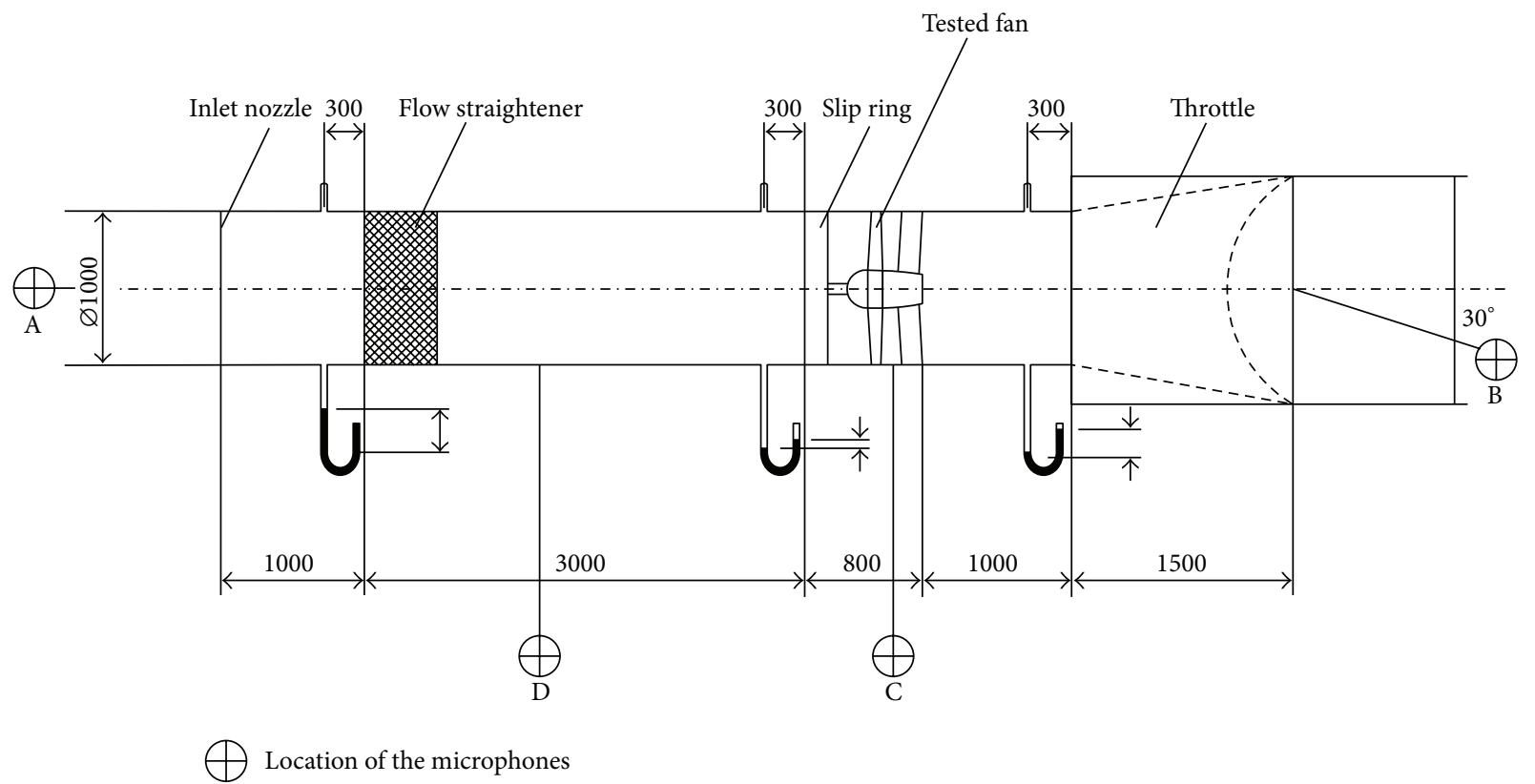

Figure 2: Test rig and measurement setup (not to scale).
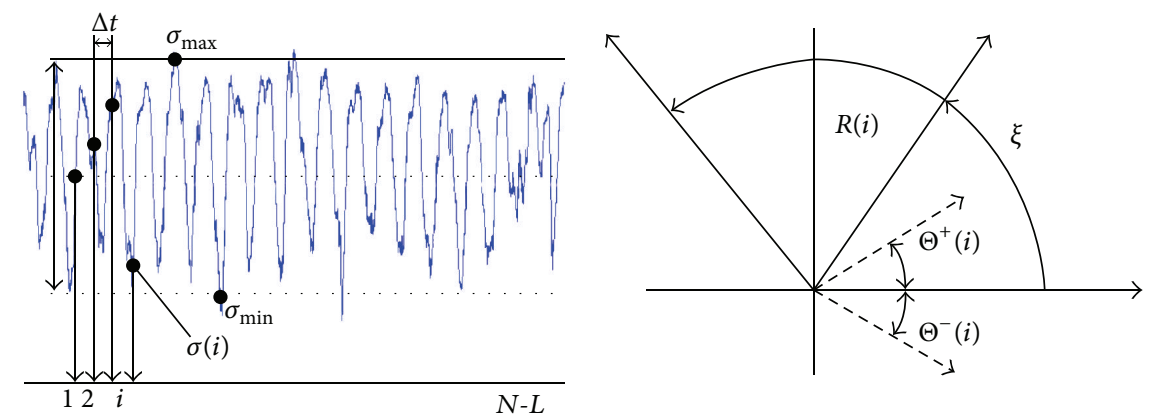

FIGURE 3: Schematic diagram of technique for plotting SDP.

number of points in a data series (determined by the sampling frequency and the sampling time) on the SDP patterns features. This paper aims to detect aerodynamic instabilities as a stall precursor. As a consequence of the short time available to detect aerodynamic instability, establishing the minimum sampling frequency is a key factor in the successful application of the SDP methodology. Drawing on previous studies using this technique [14], the SDP-based approach proved effective in a range of time windows from 0.1 to $0.004 \mathrm{~s}$. This finding confirmed the SDP potential technique as a diagnostic tool for rotating instabilities related to stall inception in fair time lag. The time window where the authors applied the SDP is clearly dependent on the rotor characteristic speed and, looking at the investigated lowspeed fan specifications, the authors chose it at $0.1 \mathrm{~s}$ (one-half rotor revolutions).

3.2. Signal Characterisation. Microphones were used to resolve high-frequency noise signals whilst maintaining good sensitivity. The far-field pressure signal was recorded at a sampling rate of $10 \mathrm{kHz}$ and considered the Nyquist frequency as $f=5 \mathrm{kHz}$. The resulting Nyquist rate was largely below the sampling frequency, thus verifying the second condition in the Nyquist problem on signal aliasing. The estimated overall uncertainties on unsteady pressure measurements were as follows: (i) $\Delta V=1000 \mathrm{mV} \pm 12 \mathrm{mV}(20: 3)$ on the voltage and (ii) $\Delta G=200 \mathrm{~dB} \pm 2.4 \mathrm{~dB}(20: 3)$ on the raw signal gain [31].

\section{Results}

The noise traces as recorded in the far-field were analysed using the proposed SDP technique. In particular, the analysis covers a range of operating point which spans from neardesign condition (point $\mathrm{P}_{1}$ ) to full stall (point $\mathrm{P}_{30}$ ) through the peak pressure operation (point $\mathrm{P}_{25}$ ). The authors recognised $\mathrm{P}_{20}$ by the noise pressure visual inspection as the stall incipience point [32]. Figure 4 shows the fan's operating interval adopted in the present discussion, plotted against the typical noise signals which the authors recorded in the inlet location (A). 


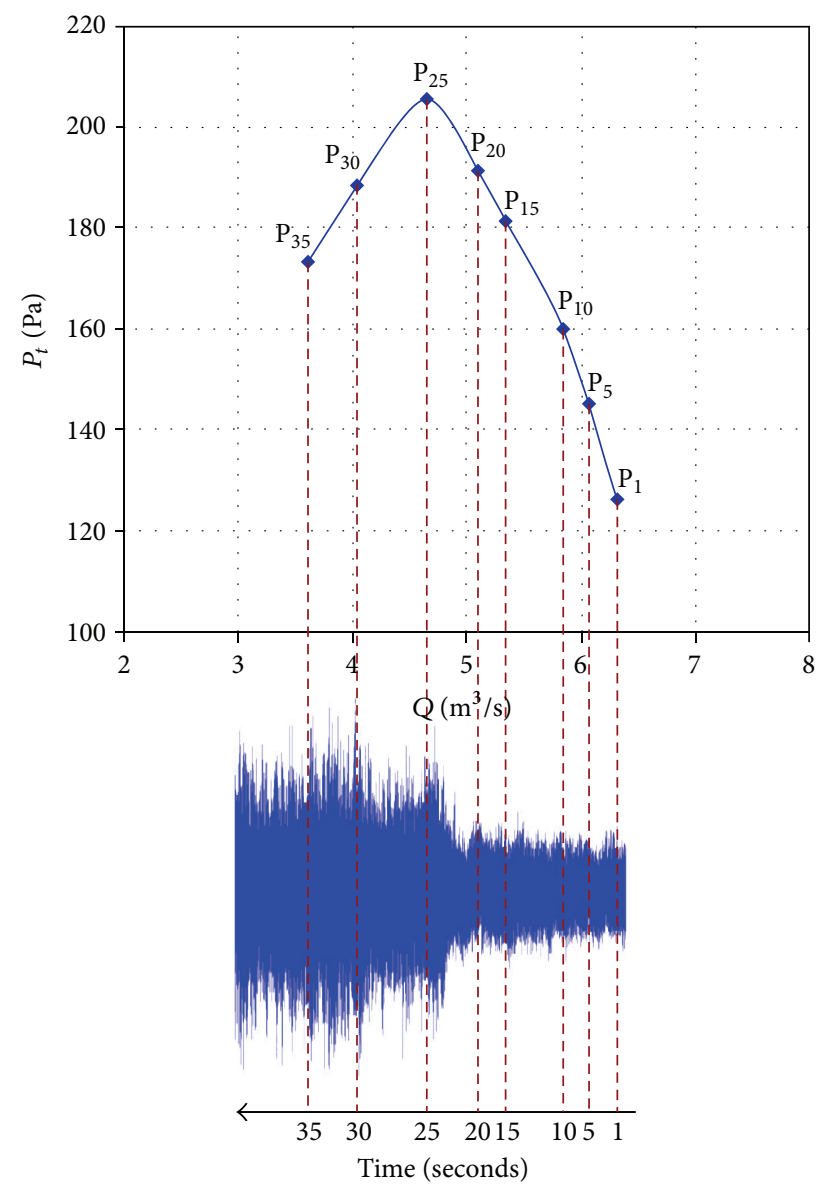

FIGURE 4: Fan investigated operating points and noise pressure trace time history.

TABLE 2: Sensitivity test matrix.

\begin{tabular}{lcc}
\hline Type of test & Constants & Parameters \\
\hline Sensitivity to angular gain & $\begin{array}{c}L=1 \\
T_{\mathrm{SDP}}=0.1 \mathrm{~s}\end{array}$ & $\xi=1,30,50$ \\
\hline Sensitivity to time lag & $\begin{array}{c}\xi=10 \\
T_{\mathrm{SDP}}=0.1 \mathrm{~s}\end{array}$ & $L=1,10,50$ \\
\hline
\end{tabular}

4.1. Sensitivity Analysis. The analyses which the authors carried out on instantaneous noise measurements had enabled the detection of safe and stalled operation regions. First SDP patterns were derived in order to give hints on the sensitivity of the mapping to the transformation metric. Table 2 describes the matrix of the tests on the inlet noise signals (A). Notably, the sampling frequency was kept constant at $10 \mathrm{kHz}$ and indicated the SDP time window $\left(T_{\mathrm{SDP}}\right)$ in seconds.

For the sake of clarity, Figures 5 and 6 show one-axis segment SDP. First, Figure 5 illustrates the sensitivity to the time lag $L$ and angular gain $\xi$ of the SDPs of the noise signals recorded at location $A$ at the normal operating conditions $\mathrm{P}_{1}$. The template patterns in Figure 5 represent the variation of the signal topology when modifying the magnitude of the time delay and angular gain which the authors used to sample it during the standard operations of the fan. For the sake of the clarity in the illustration of the sensitivity analysis, the SDP patterns are deliberately shown in one-axis plots.

Figure 6 illustrates the sensitivity to the same SDP quantities during the deep rotating stall. The dot patterns in Figure 6 illustrate the signal topology's variation when varying the $L$ and $\xi$ parameters during the stalled operations $\mathrm{P}_{35}$. Again, in order to better illustrate the evidences of the sensitivity analysis, the SDP patterns are deliberately shown in one-axis plots.

The comparison of the maps in Figure 5 allows the statement that the low time lag is more sensitive to the SDP dynamics in curvature, whilst the high $L$ values condition the SDP in a quasi-rectilinear shape. On the contrary, the high angular gain appeared richer in the dots distribution and this might allow to resolve minimal signal perturbation [33]. The sensitivity on the change of operative points drops off the comparison of Figures 5 and 6.

As expected, the angular gain exerts a critical role in the reconstruction of the pressure dynamics when throttling the fan. The patterns in Figures 5 and 6 confirm that the use of small gain is often unable to resolve the signal's modification, even in the presence of large fluctuations. For instance, with $\xi=1$, moving from $P_{1}$ to $P_{35}$ operation the mapping does not sense curvature (frequency) changes inside the main signal, but only the reduction of the normalised magnitude. For this reason, the authors chose to use a low time lag value $(L=10)$ and a high angular gain value $(\xi=60)$ in the SDP construction of the present work. The use of high angular gain parameter, as Figures 5 and 6 show, gives rise to the signal's wider polar images, due to the spreading of the points pattern. At the same time, the use of a low time lag sensed the polar plot to any changing in frequency hidden inside the fan noise signal.

This circumstance might better contribute to the capability of the SDP technique to discriminate the stable operation $\left(\mathrm{P}_{1}\right.$ point $)$ from any operative point at the time that a rotating instability might appear, which correlates with a dominant curvature and frequency presence. Note that the optimal values of $L$ and $\xi$ are strictly dependent on the fan's particular aerodynamics where the authors apply SDP analysis. Any change in the fan operative characteristics (pitch, rotor speed, etc.), or in its geometry and dimensions, would see a different tuning of the parameters in this diagnostic system.

4.2. Detection of the Stall Incipience. Indeed, the possibility of discriminating from normal operations to abnormal (also when using one-half rotor revolutions) is a key factor in the development of an effective early warning technique. Figure 7 shows how the template comparison clearly differentiated along the throttle line and between the two SDPs at normal $\left(\mathrm{P}_{1}\right)$ and aerodynamically abnormal operations $\left(\mathrm{P}_{20}, \mathrm{P}_{25}\right)$ in the different locations. As such, the criterion based on which the stall detection relies could be the visual matching of the patterns, with respect to existing templates, that can play the basic role in a control system [24].

From a first glance at the Figure 7 maps, one can dissect two major modifications when looking at their evolution 

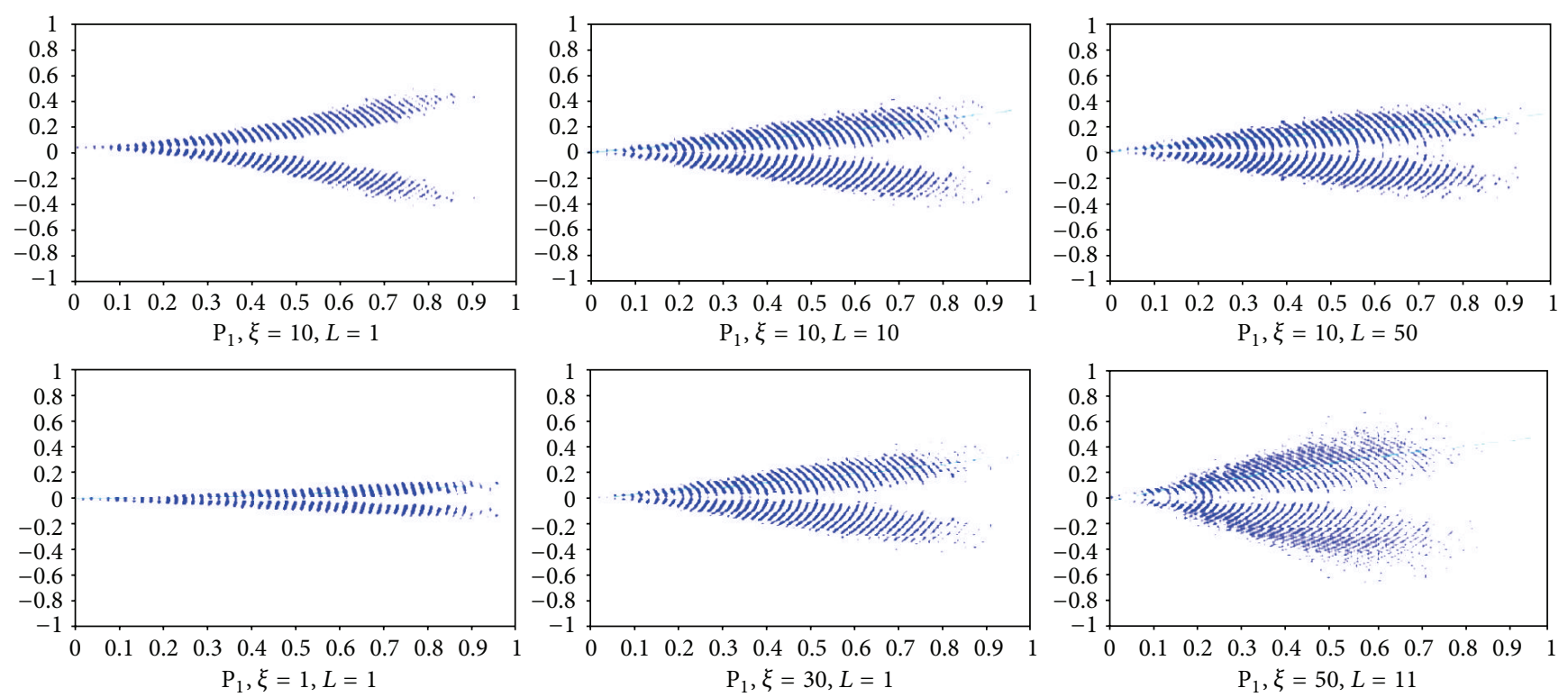

Figure 5: Sensitivity of $L$ and $\xi$ at $\mathrm{P}_{1}$.
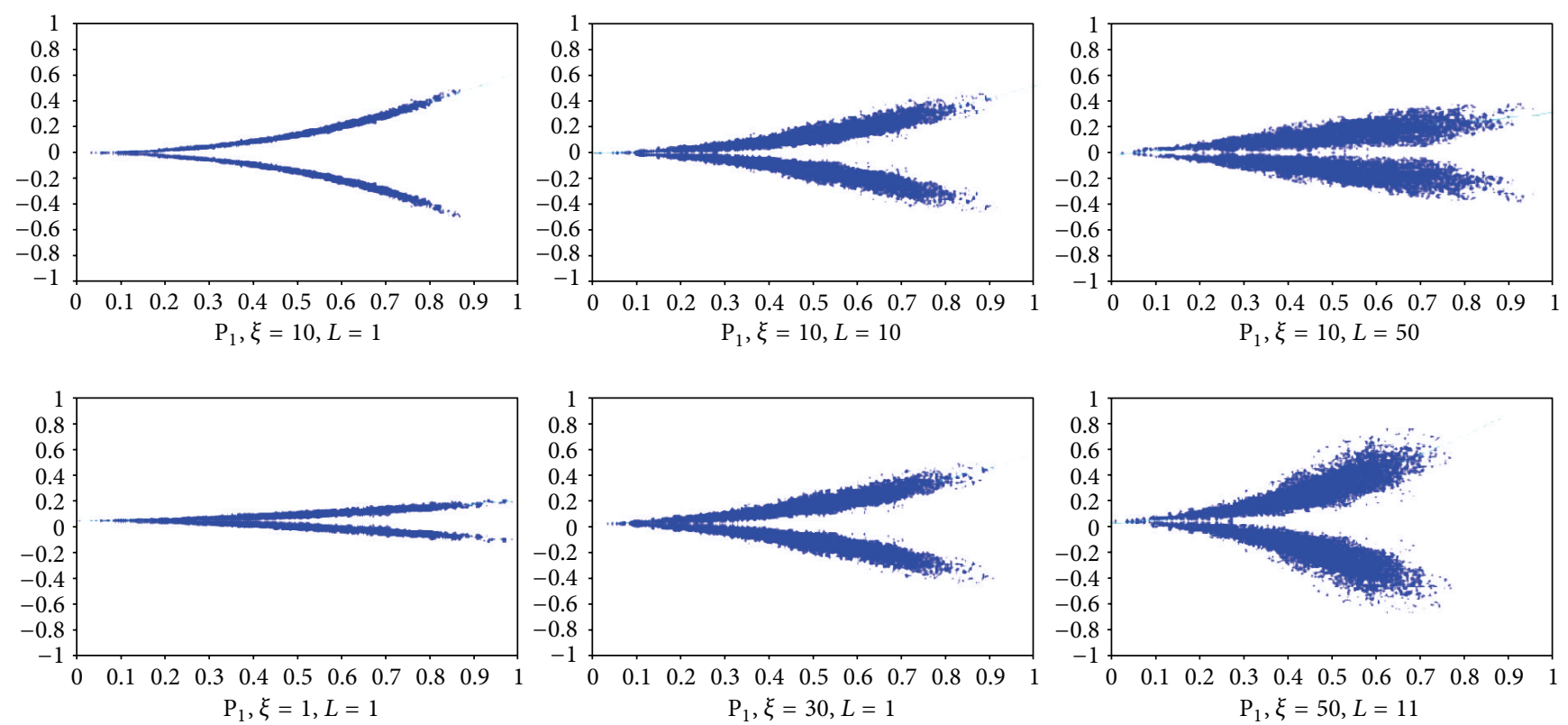

FIGURE 6: Sensitivity of $L$ and $\xi$ at $\mathrm{P}_{35}$.

toward the stalled operations (from top to bottom). First, the evidence that the pattern arms are gradually acquiring their curvature signifies the acceleration of the low-frequency components that creates the conditions for the rotating stall operation [14]. Second, the footprint in the wide SDP map at normal operations reduced with the appearance of increasing instability in the noise time history toward the full stall under the leading influence of low-frequency signal modulations driven by the rotating stall. With respect to any considered microphone locations, all the tests showed the ability to detect in the recorder signals the precursors of stall at least $5 \mathrm{~s}$ prior to the fan pressure peak $\left(\mathrm{P}_{25}\right)$ and the full stalled condition $\left(\mathrm{P}_{30}\right)$. This circumstance was always obtained by keeping the sampling time of the SDP analysis at $0.1 \mathrm{~s}$, that is, 1.5 rotor revolutions. Figure 4 provides the time history. The fastest detection was for location $\mathrm{C}$, which was able to detect the changing due to the stall incipience $10 \mathrm{~s}\left(\mathrm{P}_{15}\right)$ prior to the peak (location $\mathrm{C}$ actually sensed the modification of SDP well at $\mathrm{P}_{10}$ ). However, the particular portraits produced in location $\mathrm{C}$ were unable to follow the fan operation steps from $\mathrm{P}_{15}$ to $\mathrm{P}_{25}$, as its SDP shape did not appreciably change in this pressure range. 

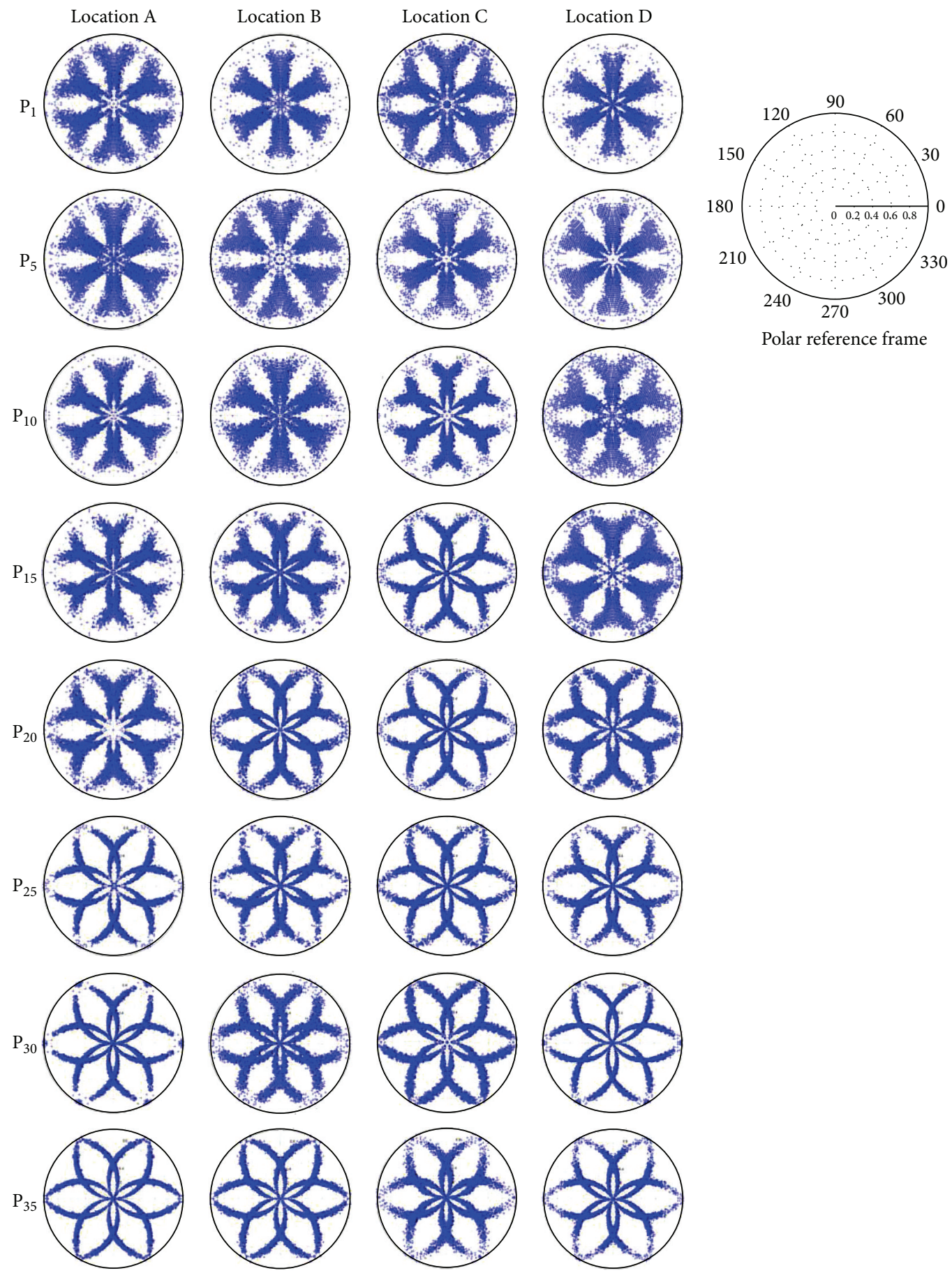

FIGURE 7: SDP evolution from stable to full stall at different locations.

The next section discusses questions which arose on this topic and the authors' attempt to provide answers.

\section{Comments on the Different Microphone Locations}

The microphone locations did not seem to influence stall condition prediction. The authors noticed few differences in the comparison to the SDP from the different locations. For facilitating the analysis of the phenomena which might play a role in the SDP portrait construction, the authors analysed in detail the SDP maps at $\mathrm{P}_{1}, \mathrm{P}_{15}$, and $\mathrm{P}_{25}$ in the locations $\mathrm{A}$ and $\mathrm{C}$. Figure 8 shows the maps.

The different SDP portrait shapes in Figure 8 show a peculiar geometry that we can relate to the chaos theory $[20,34-36]$. The cited works stress that the fractal of a random time series is usually clustered in subtriangular regions and it does not define any particular structure. By contrast, in the chaotic time series, the points are disposed 

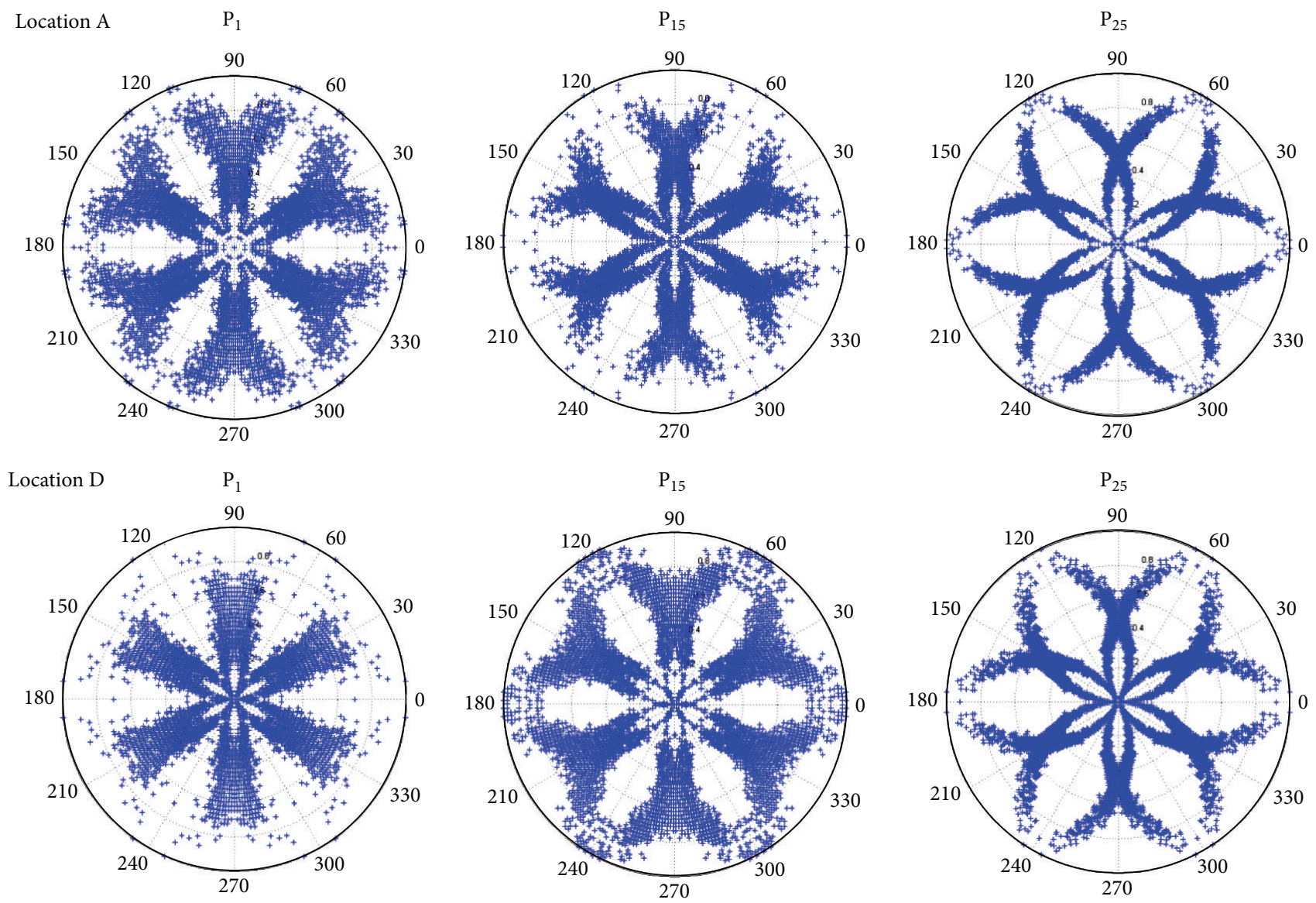

Figure 8: SDP maps at locations $\mathrm{A}$ and $\mathrm{D}$ for fan running at $\mathrm{P}_{1}, \mathrm{P}_{15}$, and $\mathrm{P}_{25}$.

along hyperbolic curves that intersect each other, indicating a clear and well-recognisable structure of the time series. Regarding the deterministic time series, the graph usually appears highly ordered with curves defining into the spread pattern that resembles petal arrangements in a flower.

Graphs in Figure 8 are quite different from those that occur in the two limit situations of purely random or deterministic time series, as an indirect evidence of a mix of structures analogous to those that the authors observed for the chaotic time series with deterministic series. The presence of so-called deterministic chaos patterns is typical of the natural time series, which characterizes the natural aero/thermodynamic phenomena [20]. We can speculate that once the perturbations responsible for the stall incipience rise, the chaotic contribution to the time series becomes more prominent and the SDP reveals its presence inside the fan noise emission. In this respect, the microphone locations could prove helpful in magnifying and isolating better the particular signature which carries the prestall information. Location D, for instance, showed a well-organised pattern with a clear deterministic shape in $\mathrm{P}_{1}$ and $\mathrm{P}_{15}$, whilst location $A$ at $P_{15}$ changed the shape to a geometry closer to a chaotic time series portrait. When comparing the diverse probe locations (i.e., A and D) at constant operating conditions (i.e., at $\mathrm{P}_{15}$ ), one can assume that there should be some advantage in the transversal duct modes that helps to isolate clearly the prestall instability's chaotic signal in the noise. On a similar fashion, it is possible to infer that the radial modes, which radiate mainly on the duct walls, are not influential. Once the stall onset is close (peak point $\mathrm{P}_{25}$ ), the SDP maps show the characteristic geometrical features of a chaotic time series which dominate irrespective from the microphone locations.

From the perspective of the industrial implementing of fan stall warning systems, use of acoustic pressure measurements is preferable, since the probe can be remotely located and thus record the acoustic signals for the fan's operating state. The measured signal pattern as input will be analysed into a template-matching system that forms a part of the data recording and analysis system. The pattern is compared to the library, with a matching system used to find the best matching from the available patterns. The "best match" pattern represents the actual operative condition, thus enabling the control system to act properly. Taking into account the complete measuring chain [14], the estimated time for pressure signal conversion and SDP processing ranges from $100 \mathrm{~ms}$ to $10 \mathrm{~ms}$ according to the data converter technology.

\section{Concluding Remarks}

The work assessed a novel diagnostic methodology for early stall detection in cooling axial-flow fans. The rationale of the proposed warning technique is to link topology and 
stall dynamics in order to discriminate between stable and unstable operations, not quantitatively but by representing howling and modulated sound components. The authors based the proposed diagnostic technique on a noise signal's polar transformation (SDP) with a constant delay time, called time lag, in a similar fashion to the phase-space portrait reconstruction method.

The SDP creates the portraits of time-discrete phase planes that occur with an axial cooling fan's cyclic system. The resulting trajectories are mathematically of interest and reveal a visually striking and intricate class of patterns ranging from stable points to stable cycle hierarchies. Their shape is linked to apparently random fluctuations which have evolved inside the main signal. The SDP system's primary focus is on the fast characterisation of simple cyclic systems using an interactive graphics system with a variety of controlling parameters.

Based on past works which used this technique, it is possible to affirm that, in the SDP of the random time series, points are usually clustered in subtriangular regions and, with the exception of the imposed sixfold symmetry which the authors used in the method, they do not define any particular structure. By contrast, in the chaotic time series, the points are disposed along hyperbolic curves that intersect each other, indicating a clear and well-recognisable time series structure (as for the SDP in stalled conditions). Regarding the deterministic time series, the graph appears highly ordered with curves defining a spread pattern that resemble a petal arrangement in a flower (with similar shape to the one for the normal operations). Indeed, the graph shapes for the investigated fan at normal operation are quite different from those that occur in the random and the deterministic series, evidencing also structures analogous to those which the authors observed for the chaotic time series. This pattern is typical of the so-called natural time series. The authors observed similar structures for all the recorded signals with superposition of a deterministic base (driven by the blade rotation) and a chaotic seeding (driven by the aerodynamic instabilities). When the perturbations responsible for the stall incipience rise, the chaotic time series becomes more prominent and the SDP reveals its presence inside the mainly natural fan noise emission time series.

This analysis indicates a strict similarity between the chaotic and natural time series suggesting the presence of chaotic dynamics in action during the fan's throttling. The SDP sensitivity to this dynamic variation does not suffer from the particular microphone locations. However, the duct modes might be helpful in amplifying those acoustic structures more sensitive to the aerodynamic instabilities' chaotic dynamics.

The results demonstrated that the proposed algorithm is able to effectively diagnose early stall signs. In particular, SDPs effectively detect nonconformity in the noise waves when disturbances are slightly higher than the background signals and are chaotic in nature. The paper proves the possibility of discriminating the presence of pressure instabilities (at stall incipience or rotating stall) also using 1.5 rotor revolutions sampling time. This circumstance confirmed the potential of the proposed approach in the definition of an effective early warning technique and implementing active stall control systems.

\section{References}

[1] G. R. Ludwig and J. P. Nenni, "A rotating stall control system for turbojet engines," ASME paper 76-GT-115, 1976.

[2] I. J. Day, T. Breuer, J. Escuret, M. Cherrett, and A. Wilson, "Stall inception and the prospects for active control in four highspeed compressors," Journal of Turbomachinery, vol. 121, no. 1, pp. 18-27, 1999.

[3] B. Höss, D. Leinhos, and L. Fottner, "Stall inception in the compressor system of a turbofan engine," Journal of Turbomachinery, vol. 122, no. 1, pp. 32-44, 2000.

[4] S. Bianchi, A. Corsini, and A. G. Sheard, "Stall inception, evolution, and control in a low speed axial fan with variable pitch in motion," Journal of Engineering for Gas Turbines and Power, vol. 134, Article ID 042602, 10 pages, 2012.

[5] J. T. Gravdahl and O. Egeland, Compressor Surge and Rotating Stall: Modeling and Control, Springer, London, UK, 1999.

[6] N. A. Cumpsty, "Part-circumference casing treatment and the effect on compressor stall," ASME paper 89-GT, 1989.

[7] F. K. Moore, "A theory of rotating stall of multistage compressors, parts I-III," ASME Journal of Engineering for Power, vol. 106, pp. 313-336, 1984.

[8] A. Rippl, Experimentelle Untersuchungen zuminstationaren Betriebsverhahen an der Stabilitarsgrenze eines mehrstufigen trans-sonischen Verdichters [Ph.D. thesis], Ruhr-Universitat Bochum, 1995.

[9] A. G. Sheard and A. Corsini, "The impact of an anti-stall stabilisation ring on industrial fan performance: implications for fan selection," in Proceedings of the 55th American Society of Mechanical Engineers Turbine and Aeroengine Congress, paper no. GT2011-45187, Vancouver, Canada, June 2011.

[10] J. D. Paduano, E. M. Greitzer, and A. H. Epstein, "Compression system stability and active control," Annual Review of Fluid Mechanics, vol. 33, pp. 491-517, 2001.

[11] Y. Liu, M. Dhingra, and J. V. R. Prasad, "Active compressor stability management via a stall margin control mode," ASME paper GT2009-60140, 2009.

[12] S. Bindl, M. Stö $\beta$ el, and R. Niehuis, "Stall detection within the low pressure compressor of a twin-spool turbofan engine by tip flow analysis," ASME paper GT2009-59032, 2009.

[13] F. O. Methling, H. Stoff, and F. Grauer, "The prestall behavior of a 4-stage transonic compressor and stall monitoring based on artificial neural networks," International Journal of Rotating Machinery, vol. 10, pp. 387-399, 2004.

[14] A. G. Sheard, A. Corsini, and S. Bianchi, "Stall warning in a low-speed axial fan by visualization of sound signals," Journal of Engineering for Gas Turbines and Power, vol. 133, no. 4, Article ID 041601, 2011.

[15] M. Tryfonidis, O. Etchevers, J. D. Paduano, A. H. Epstein, and G. J. Hendricks, "Prestall behavior of several high-speed compressors," Journal of Turbomachinery, vol. 117, no. 1, pp. 6280, 1995.

[16] D. Christensen, P. Cantin, D. Gutz et al., "Development and demonstration of a stability management system for gas turbine engines," ASME paper GT2006-90324, 2006. 
[17] Z. Tong, L. Li, C. Nie, B. Lin, Y. Cui, and W. Qi, “On-line stall control with the digital signal processing method in an axial compressor," ASME paper GT2009-59509, 2009.

[18] N. Tahara, M. Kurosaki, Y. Ohta, E. Outa, T. Nakajima, and T. Nakakita, "Early stall warning technique for axial-flow compressors," Journal of Turbomachinery, vol. 129, no. 3, pp. 448-456, 2007.

[19] F. A. E. Breugelmans, C. Palomba, and T. Funk, "Application of strange attractors to the problem of rotating stall," in Unsteady Aerodynamics and Aeroelasticity in Turbomachinery, Y. Tanida and M. Namba, Eds., Elsevier Science, 1995.

[20] F. Takens, "Detecting strange attractors in turbulence," in Lecture Notes in Mathematics, D. A. Rand and L. S. Young, Eds., Springer, Berlin, Germany, 1981.

[21] M. M. Bright, E. Qammar, H. Vhora, and M. Schaffer, "Rotating pip detection and stall warning in high-speed compressors using structure function," in Proceedings of the RTO AVT Symposium, Toulouse, France, May 1998.

[22] K. Shibata, A. Takahashi, and T. Shirai, "Fault diagnosis of rotatine machinery through visualisation of sound signals," Mechanical Systems and Signal Processing, vol. 14, pp. 229-241, 2000.

[23] J. Wu and C. Chuang, "Fault diagnosis of internal combustion engines using visual dot patterns of acoustic and vibration signals," NDT\&E International, vol. 38, pp. 605-614, 2005.

[24] A. G. Sheard, A. Corsini, and S. Bianchi, "Method of detecting stall in an axial fan," British Patent application no. P 10896 GB, application date 1 March, 2010.

[25] S. Bianchi, A. Corsini, F. Rispoli, and A. G. Sheard, "Detection of aerodynamic noise sources in low-speed axial fans with tip endplates," Proceedings of the Institution of Mechanical Engineers, Part C: Journal of Mechanical Engineering Science, vol. 223, no. 6, pp. 1379-1392, 2009.

[26] C. A. Pickover, "On the use of symmetrized dot patterns for the visual characterization of speech waveforms and other sampled data," The Journal of the Acoustical Society of America, vol. 80, no. 3, pp. 955-960, 1986.

[27] T. J. Schultz, "Synthesis of social surveys on noise annoyance," Journal of the Acoustical Society of America, vol. 64, pp. 377-405, 1978.

[28] R. Sottek and K. Genuit, "Sound quality evaluation of fan noise based on hearing-related parameters," in Proceedings of the Fan Noise 3rd International Symposium, Lyon, France, 2007.

[29] E. M. Greitzer, "Surge and rotating stall in axial flow compressors, part I: theoretical compression system model," ASME Journal of Engineering for Power, vol. 98, pp. 190-198, 1976.

[30] J. Gleick, Chaos: Making a New Science, Vintage Books, 1987.

[31] H. L. Boerrigter, PreMeSys: A Simulation Program to Determine the Frequency and Time Response of a Pressure Measurement System, VKI Technical Memorandum, von Karman Institute for Fluid Dynamics, 1996.

[32] T. Arts, H. Boerrigter, M. Carbonaro et al., Measurement Techniques in Fluid Dynamics-An Introduction, von Karman Institute for Fluid Dynamics, Rhode Saint Genèse, Belgium, 2009.

[33] B. De-Rosier, M. D. Normand, and M. Peleg, "Effect of lag on the symmetrised dot pattern (SDP) displays of the mechanical signatures of crunchy cereal foods," Science of Food and Agriculture, vol. 75, pp. 173-178, 1997.

[34] C. A. Pickover, Computers, Pattern, Chaos and Beauty, St Martin's Press, New York, NY, USA, 1990.
[35] A. M. Fraser and H. L. Swinney, "Independent coordinates for strange attractors from mutual information," Physical Review A, vol. 33, no. 2, pp. 1134-1140, 1986.

[36] D. Perugini, T. Busà, G. Poli, and S. Nazzareni, "The role of chaotic dynamics and flow fields in the development of disequilibrium textures in volcanic rocks," Journal of Petrology, vol. 44, no. 4, pp. 733-756, 2003. 

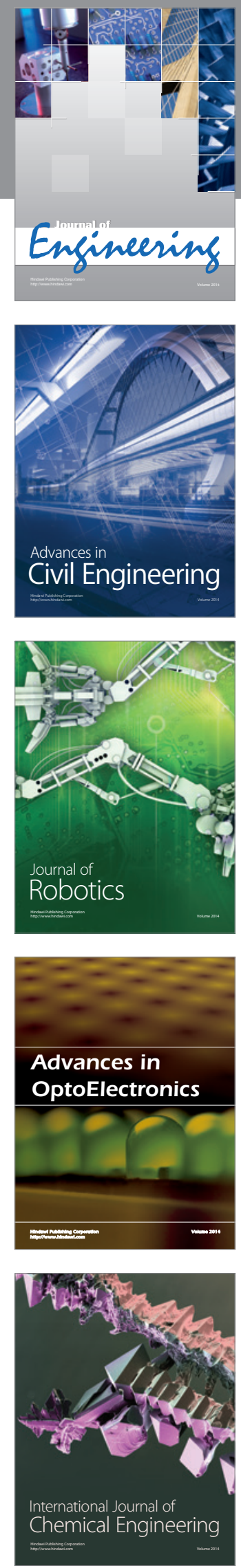

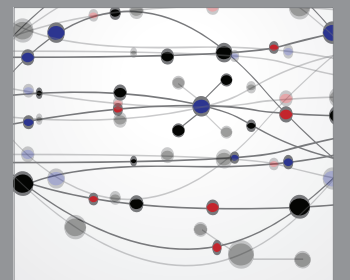

The Scientific World Journal
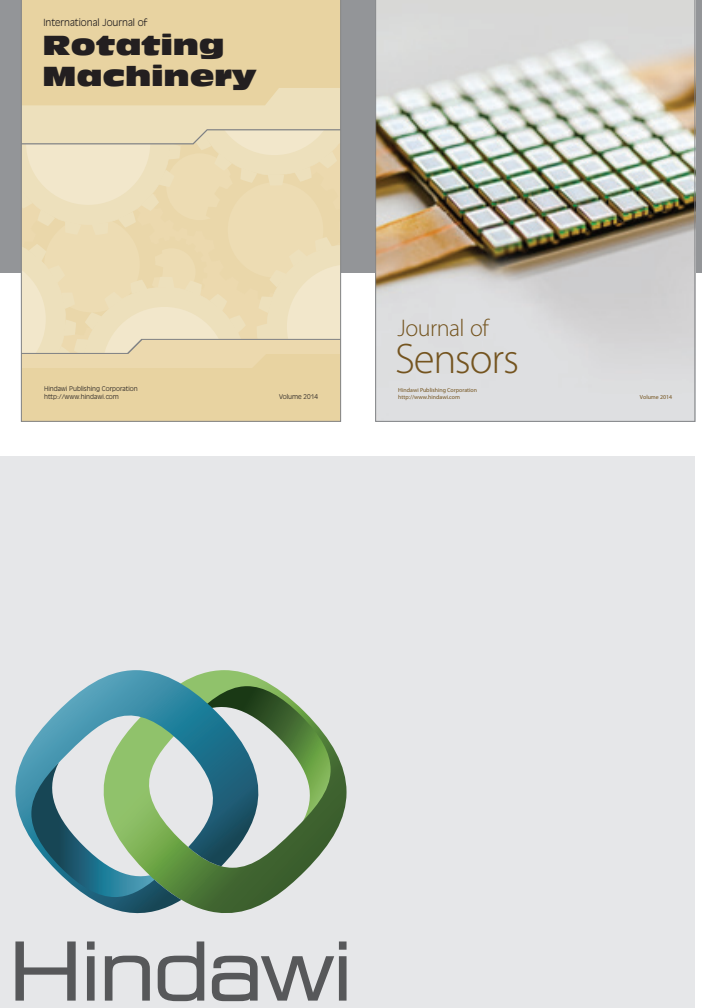

Submit your manuscripts at http://www.hindawi.com
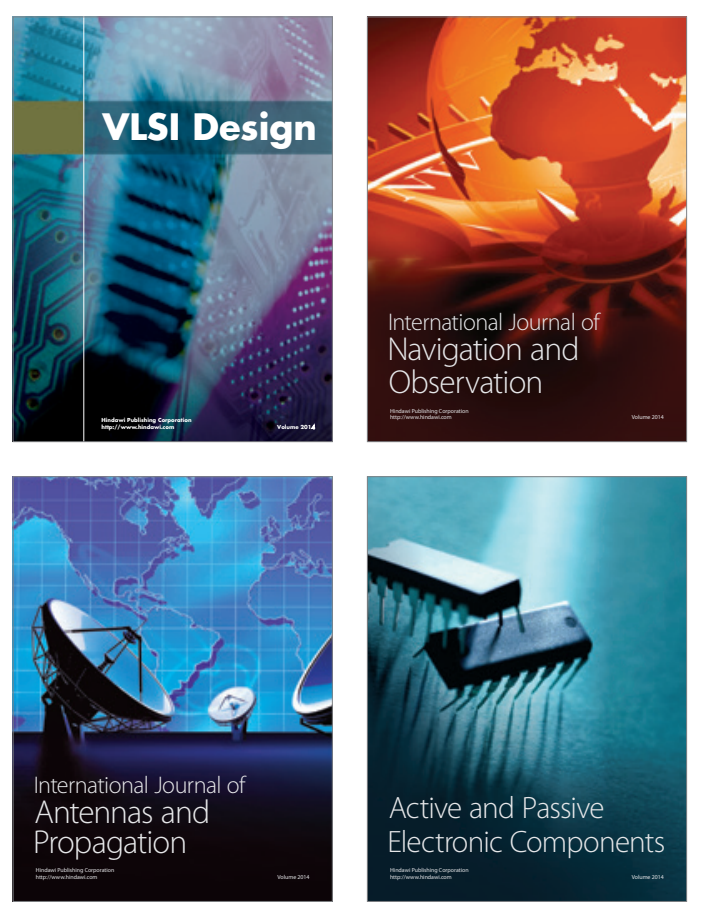
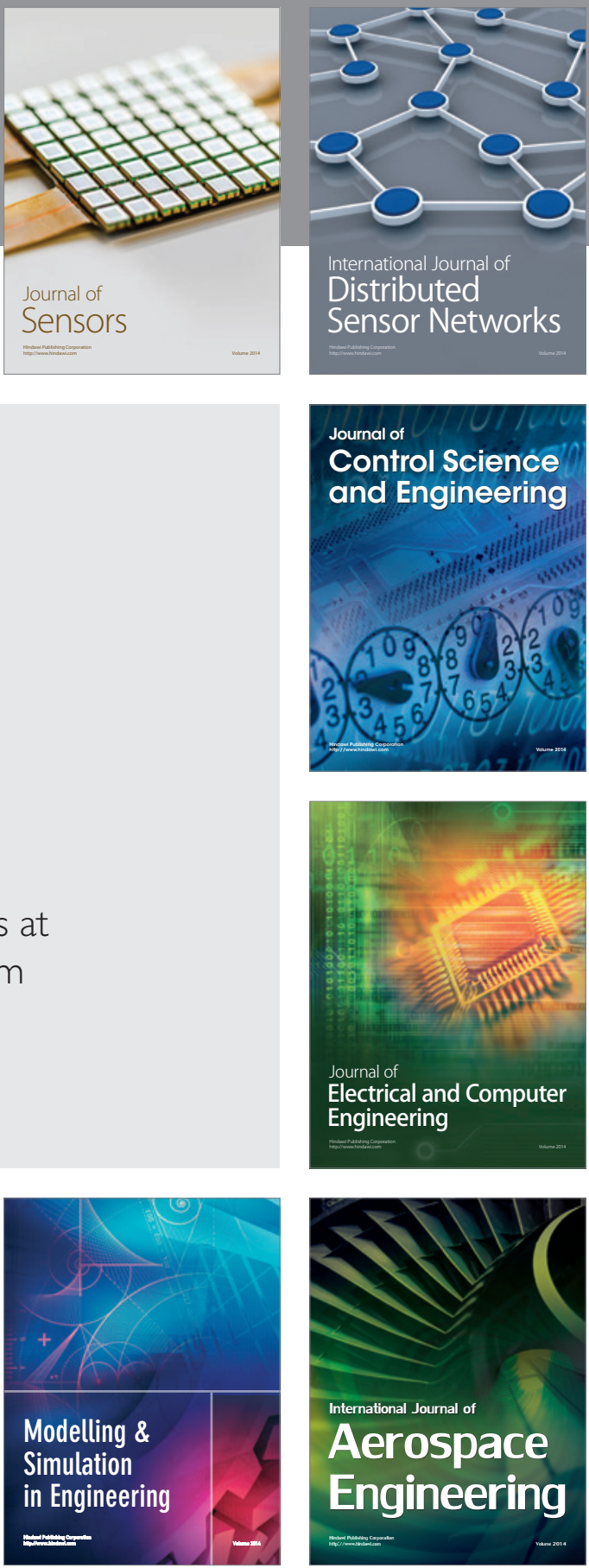

Journal of

Control Science

and Engineering
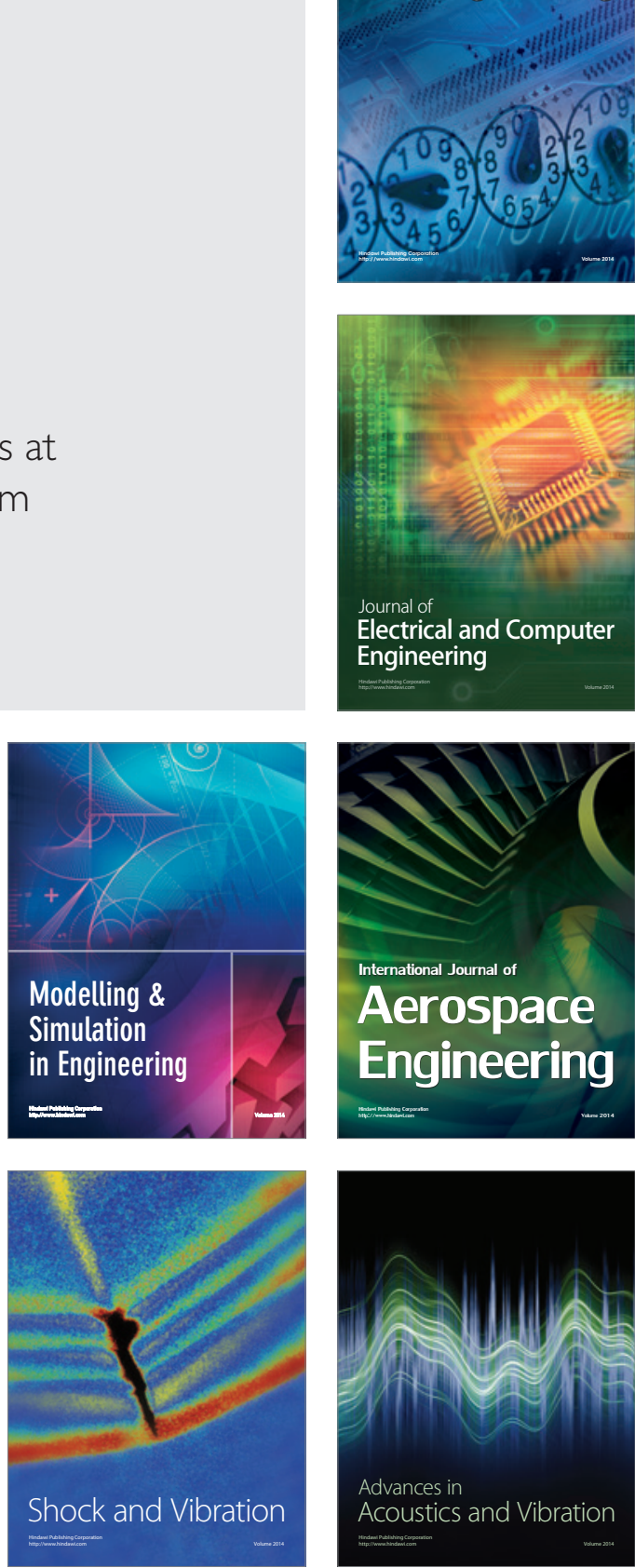